\title{
Newly constructed compact accelerator-based neutron facility at AIST
}

\author{
Koichi Kino ${ }^{1,2,}$, Takeshi Fujiwara ${ }^{1,2}$, Michihiro Furusaka ${ }^{1,2}$, Noriyosu Hayashizaki ${ }^{1,2,3}$, Hidetoshi Kato $^{1,2}$, \\ Ryunosuke Kuroda ${ }^{1,2}$, Koji Michishio ${ }^{1,2}$, Takemi Muroga ${ }^{2}$, Hiroshi Ogawa ${ }^{1,2}$, Brian E. O'Rourke ${ }^{1,2}$, \\ Nagayasu Oshima ${ }^{1,2}$, Daisuke Satoh ${ }^{1,2}$, Norihiro Sei ${ }^{1,2}$, Tamao Shishido ${ }^{2}$, Ryoichi Suzuki ${ }^{1,2}$, Masahito Tanaka ${ }^{1,2}$, \\ Yo Tomota ${ }^{1,2}$, Hiroyuki Toyokawa ${ }^{1,2}$, Akira Watazu ${ }^{1,2}$, Kazuro Furukawa $^{1,4}$, Kazuyuki Nigorikawa ${ }^{1,4}$, and Takashi Obina ${ }^{1,4}$ \\ ${ }^{1}$ National Institute of Advanced Industrial Science and Technology (AIST), Tsukuba, Ibaraki, Japan \\ ${ }^{2}$ Innovative Structural Materials Association (ISMA), Chiyoda-ku, Tokyo, Japan \\ ${ }^{3}$ Tokyo Institute of Technology (Tokyo Tech), Meguro-ku, Tokyo, Japan \\ ${ }^{4}$ High Energy Accelerator Research Organization (KEK), Tsukuba, Ibaraki, Japan
}

\begin{abstract}
We have constructed a compact accelerator-based neutron facility at the National Institute of Advanced Industrial Science and Technology (AIST) in Japan aiming at development of structural materials of transportation vehicles for weight reduction. The facility consists of electron linear accelerator, neutron source, and a neutron beamline with a measurement hutch. Currently we are commissioning the accelerator and expecting a first neutron beam in 2019.
\end{abstract}

\section{Introduction}

An energy-saving society is required recently and regulations of $\mathrm{CO}_{2}$ exhaust are getting tighter. One of the solutions is reducing weight of transportation vehicles like automobiles by "multi-material design". Multimaterial design involves choosing and applying lightweight materials, such as high strength steel, aluminum or carbon-fiber reinforced plastic (CFRP). The key is the joining technology of these dissimilar materials. For practical realization of such designs, it is important to analyze not only test pieces but also assembled structural materials non-destructively.

A neutron beam is effective for nondestructive analysis due to its high material penetrability. A particularly useful technique for materials analysis is wavelength-resolved neutron transmission imaging. This imaging technique requires a white (i.e. a beam with a broad energy distribution) pulsed neutron beam. The neutron beam is irradiated on a sample and transmitted neutrons are detected by a position sensitive neutron detector with the time of flight (TOF) between the neutron source and the detector of each neutron measured. The wavelength of a neutron is proportional to the TOF as the wavelength is inversely proportional to the neutron's velocity. If the sample has a crystalline nature, neutron diffractions are seen in the transmission spectrum as a function of a neutron wavelength. By analyzing the transmission spectra for all neutron detection positions, macroscopic images on the crystalline information can be obtained. This method is called Bragg edge imaging. Many pioneering measurements have been reported for crystalline phase, crystalline strain, crystalline size, and crystalline orientation especially with steel samples [1-7]. Such imaging data is easily understood by people in industrial fields, who are not neutron analysis experts.

Furthermore, a compact accelerator-based neutron source (CANS) is useful for industrial usages by ondemand measurements. Therefore, we think a combination of a CANS and the wavelength-resolved neutron transmission imaging matches to our purpose. We have designed and constructed a CANS at the National Institute of Advanced Industrial Science and Technology (AIST) in Tsukuba, Japan [8]. In this paper, the main components, the expected properties of the neutron beam, and the current status of the new CANS are described.

\section{Main components}

Figure 1 shows the overview of the CANS at AIST. It mainly consists of an electron linear accelerator, neutron source, and neutron beamline.

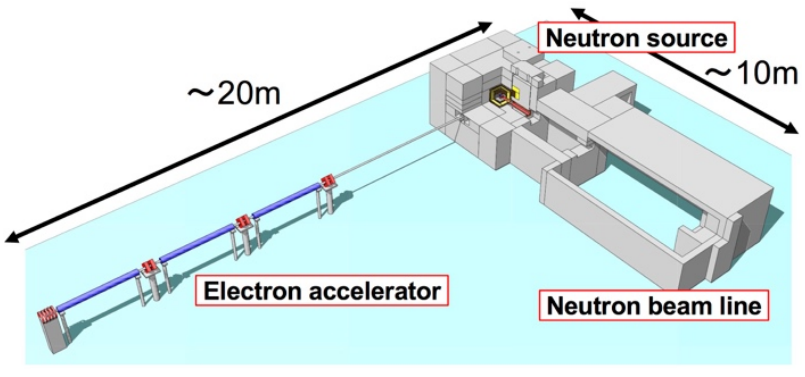

Fig. 1. Overview and features of the CANS at AIST [8].

\footnotetext{
* Corresponding author: koichi.kino@aist.go.jp
} 
An electron beam of about $35 \mathrm{MeV}$, with a maximum pulse width of $10 \mu \mathrm{s}$, and a maximum repetition rate of $100 \mathrm{~Hz}$ is produced by the electron linear accelerator [9]. The pulse width is optimized for a short pulse neutron beam by a decoupled solid methane moderator described later. The maximum repetition rate is optimized for the neutron wavelength region needed for the Bragg edge imaging. The designed maximum electron beam power is about $10 \mathrm{~kW}$ and this value is about 10 times larger than that of the HUNS (Hokkaido University Neutron Source)-I.

In the neutron source, the electron beam is irradiated on a neutron production target, which consists of several layers of thin tantalum plates with cooling water flowing between them. Evaporation neutrons generated in the tantalum plates are slowed down in a moderator positioned directly above the neutron production target. The moderator is a block of solid methane with a thickness of $3 \mathrm{~cm}$ cooled to $20 \mathrm{~K}$. The dimensions and the decoupled designs (i.e. the moderator is surrounded by cadmium except for the neutron extraction hole) were chosen for a short pulse length of the neutron beam, which is needed for a high neutron wavelength resolution due to the limited length of the flight path.

The neutron beamline is surrounded by a radiation hutch made of concrete. The flight path length is $8 \mathrm{~m}$ due to the size of the hutch which is limited by the size of the experimental room. Super mirror guide tubes of $3 Q_{\mathrm{c}}$ are set along the neutron beamline to increase the neutron flux at the sample position. The inner dimensions and total length of the guide tubes are $130 \mathrm{~mm} \times 130 \mathrm{~mm}$ and $5 \mathrm{~m}$. Furthermore, we are preparing a novel position sensitive neutron detector, which has a high detection efficiency and counting rate capability. The detector is based on gaseous detector (Glass GEM) with Boron foil neutron converter [10]. In order to improve the detection efficiency, micro-structured aluminium substrate were coated with ${ }^{10} \mathrm{~B}$ [11]. The combination of high neutron production rate, with efficient transport and detection will allow measurements with good statistics within practical measurement time.

\section{Properties of the neutron beam}

We have calculated the expected properties of the neutron beam using a Monte-Carlo simulation [8]. Figure 2 shows the neutron wavelength resolution at $\mathrm{L}$ (flight path length) $=8 \mathrm{~m}$ (i.e. at the sample position).

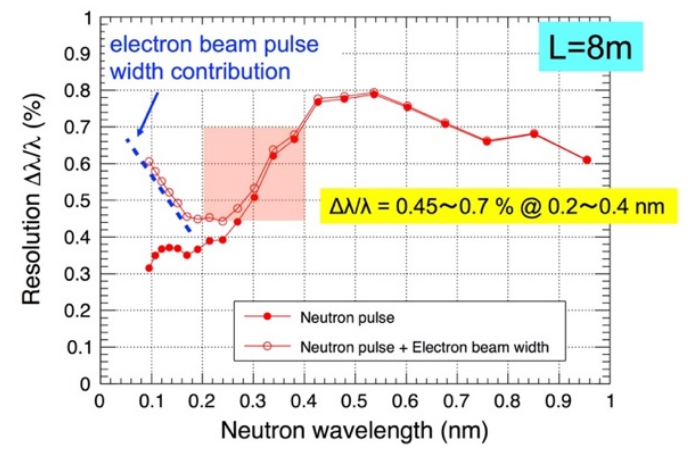

Fig. 2. Neutron wavelength resolution at $\mathrm{L}=8 \mathrm{~m}$ [8].
The maximum Bragg edge wavelength for structural materials such as steel and aluminium is about $0.4 \mathrm{~nm}$. In the wavelength region from 0.2 to $0.4 \mathrm{~nm}$ the resolution is $0.45 \sim 0.7 \%$. This value is about 3 times larger than that of the J-PARC neutron imaging beamline (BL22) [12] mainly due to a difference of the flight path lengths. The wavelength resolution deteriorates below $0.2 \mathrm{~nm}$ as the wavelength becomes shorter. This is due to the pulse width of the electron beam $(10 \mu \mathrm{s})$, which also contributes to the sharpness of the neutron beam in addition to the time width by the slowing down process in the moderator.

The neutron flux at $\mathrm{L}=8 \mathrm{~m}$ is shown in Fig. 3. The simulation includes a super mirror guide tube along the neutron beam extraction hole in the beam shutter. Super mirror guide tubes can also be placed downstream of the beam shutter, which consist of two parts (labelled upstream and downstream). We calculated the neutron flux at $\mathrm{L}=8 \mathrm{~m}$ for three configurations of the super mirror guide tubes; 1 . shutter only, 2. shutter and upstream and 3. shutter, upstream and downstream mirrors. The results show a thermal neutron peak around $0.4 \mathrm{~nm}$ and the intensity of this peak increases as the number of super mirror guide tubes is increased. In the thermal and cold neutron energy region, the calculated instantaneous maximum neutron flux ranges from $2.5 \times 10^{4} \sim 1.0 \times 10^{5} / \mathrm{cm}^{2} / \mathrm{s}$ depending on the mirror configuration. The maximum counting rate of currently available position sensitive neutron detectors, which have an effective area of $10 \mathrm{~cm} \times 10 \mathrm{~cm}$, is $10^{5} \sim 10^{6} / \mathrm{s}$. If a typical neutron detection efficiency of $10 \%$ at 0.4 $\mathrm{nm}$ is assumed, these detectors will reach maximum counting rate for a neutron beam with a flux of $10^{4} \sim 10^{5}$ $/ \mathrm{cm}^{2} / \mathrm{s}$. This indicates that the expected neutron flux of the present CANS corresponds to the maximum detection rate of existing position sensitive detectors.

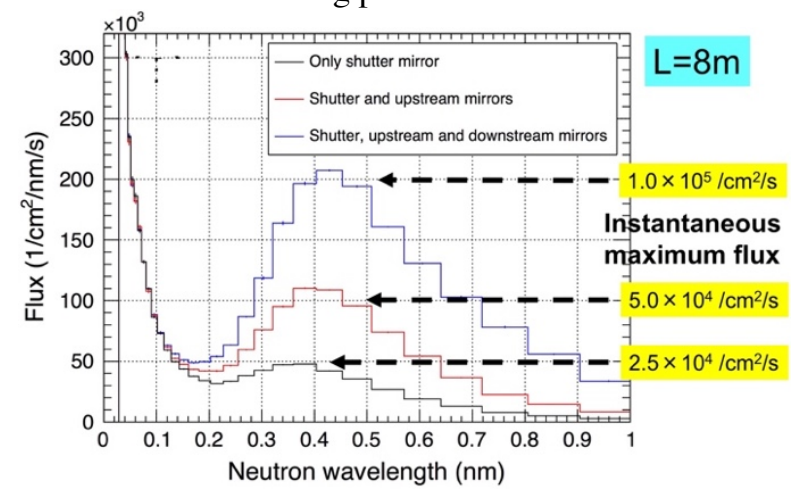

Fig. 3. Neutron flux at $\mathrm{L}=8 \mathrm{~m}[8]$.

\section{Current status of the CANS}

Figure 4 is a picture of the electron linear accelerator. An electron beam is accelerated from electron gun through three $2.7 \mathrm{~m}$ long accelerator cavities. The accelerated electron beam is then directed onto the neutron production target, which is located in an experimental room separated from the accelerator room by a $5 \mathrm{~m}$ thick concrete wall. 
Currently we are commissioning the accelerator. A beam loading of the accelerator cavity 1 was observed in an RF dummy load signal when the electron gun was tuned on. We also observed a beam spot on a screen monitor set just downstream the accelerator cavity 1 . The beam size was about $30 \mathrm{~mm}$ in diameter but focusing magnets were not used at this time.

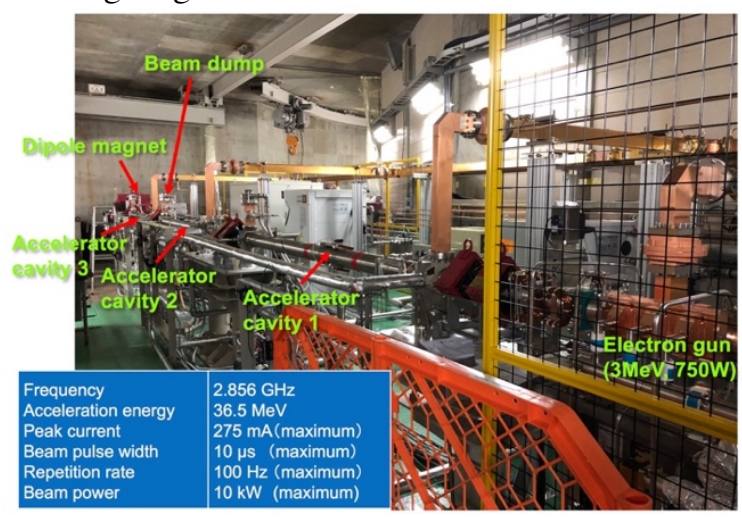

Fig. 4. Photo of the electron linear accelerator.

The electron beam comes from the electron linear accelerator in the neighboring room (to the left hand side in this picture) and is irradiated on the neutron production target shown in Fig. 5 at the center of the neutron source. A cooling water test of the target has been done. Tests of the solid methane moderator test are ongoing. Solid methane is produced by condensation of methane gas by cooling down the aluminum moderator vessel. Condensation is confirmed not only by the methane gas pressure and temperature of the vessel but also by X-ray imaging of the liquid and solid methane. Liquid and solid methane were clearly observed in X-ray images.

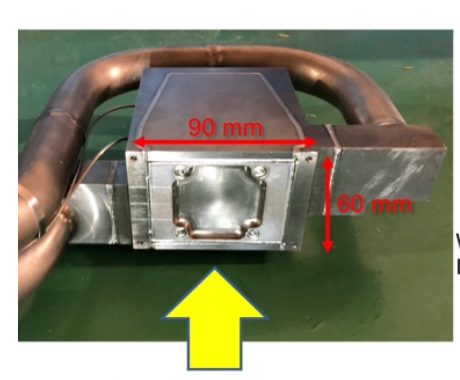

Electron beam

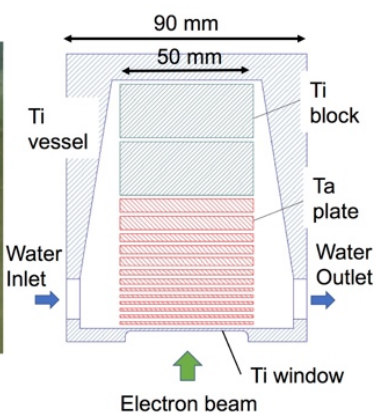

Electron beam
Fig. 5. Neutron production target.

Access into the beamline hutch is controlled by a door at the hutch entrance. The neutron beam can be blocked off using a shutter, preventing neutrons from entering the hutch when the shutter is closed. The shutter controller and door controller panel is located just outside the entrance. If the shutter is closed, the door can be unlocked and opened and workers can enter the hutch even when the accelerator is in operation. Before the beam shutter can be opened, all workers must evacuate from the hutch and the door must be locked within 20 seconds after the evacuation button is pushed. The status of the shutter, door and evacuation condition are controlled by a personal protection system. Status of the beam shutter is shown by green and red lights just above the entrance. Furthermore, this procedure is very similar to that of the J-PARC neutron beamlines. Therefore, neutron users will not feel difficulties between the present CANS and J-PARC.

Currently there are only two stages around the sample position in the hutch. We are preparing a neutron detector, sample goniometer, background shielding and so on for a first neutron beam.

\section{Summary}

We have constructed a CANS for wavelength resolved neutron transmission imaging of structural materials. The electron linear accelerator is now under commissioning. The neutron production target and solid methane moderator of the neutron source are undergoing testing. The sample position in the neutron beamline hutch is in preparation. The first neutron beam is expected later in 2019.

The new neutron facility is being developed under the auspices of the Innovative Structural Materials Association (ISMA), which is promoting weight reduction in automobiles and other transportation vehicles. This paper is based on results obtained from a project commissioned by the New Energy and Industrial Technology Development Organization (NEDO).

\section{References}

1. J.R. Santisteban, L. Edwards, M.E. Fitzpatrick, et al., Nucl. Instrum. Methods Phys. Res., Sect. A 481, 765 (2002)

2. H. Sato, T. Kamiyama, and Y. Kiyanagi, Mater. Trans., 52, 1294 (2011)

3. K. Iwase, H. Sato, S. Harjo, et al., J. Appl. Crystallogr., 45, 113 (2012)

4. Y. Su, K. Oikawa, S. Harjo, et al., Mater. Sci. Eng., A 675, 19 (2016)

5. A.S. Tremsin, S. Ganguly, S.M. Meco, et al., J. Appl. Crystallogr., 49, 1130 (2016)

6. A.S. Tremsin, T.Y. Yau, and W. Kockelmann, Strain, 52, 548 (2016)

7. K. Oikawa, Y.H. Su, R. Kiyanagi, et al., Physica B, 551, 436 (2018)

8. K. Kino, T. Fujiwara, M. Furusaka, et al., Nucl. Instrum. Methods Phys. Res., Sect. A 927, 407 (2019)

9. B.E. O'Rourke, T. Fujiwara, K. Furukawa, et al., Nucl. Instrum. Methods Phys. Res., Sect. B 464, 41 (2020)

10. T. Fujiwara, Y. Mitsuya, H. Takahashi, et al., J. Instrum. 9 P11007 (2014)

11. T. Fujiwara, U. Bautista, Y. Mitsuya, et al., Nucl. Instrum. Methods Phys. Res., Sect. A 838, 124 (2016)

12. Y. Kiyanagi, T. Kamiyama, H. Sato, et al., Nuclear Instrum. Methods Phys. Res., A 651, 16 (2011) 